\title{
Predição de casos e óbitos de COVID-19 em Mato Grosso e no Brasil
}

\section{Prediction of COVID -19 cases and deaths in Mato Grosso state and Brazil}

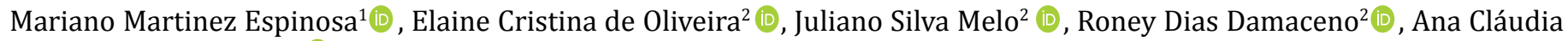 \\ Pereira Terças-Trettel ${ }^{1,3}$ (D) \\ 1.Docente do Programa de pós-graduação em Saúde Coletiva e do Departamento de Estatística da Universidade Federal de Mato Grosso (UFMT), campus \\ Cuiabá, Mato Grosso, Brasil. 2. Secretaria de Estado de Saúde de Mato Grosso (SEMT), Mato Grosso, Brasil. 3. Universidade do Estado de Mato Grosso \\ (UNEMAT), campus de Tangará da Serra, Mato Grosso, Brasil.
}

\section{Resumo}

Objetivos: prever a tendência epidemiológica de casos e óbitos do COVID-19 no Brasil e, especificamente, em Mato Grosso, por meio do modelo estatístico duplo exponencial. Métodos: para a realização deste trabalho, foram utilizados dados de casos e óbitos do COVID-19 fornecidos pelo Ministério da Saúde do Brasil e Secretaria de Estado de Saúde de Mato Grosso, e, para realizar as previsões com esses dados, foi utilizada a técnica estatística de séries temporais, considerando o modelo exponencial duplo, e para o processamento dos dados, foi utilizado o programa MINITAB V 17. Resultados: os resultados mostram que os valores ajustados pelo modelo exponencial duplo transformado dos casos e óbitos estão muito próximos dos valores observados de COVID-19 no Brasil e, principalmente, em Mato Grosso, no período considerado, indicando que esse modelo é adequado para os dados em estudo, o qual foi verificado, prevendo alguns valores com seus respectivos intervalos de confiança de $95 \%$. Conclusões: a possibilidade de se estimar, pautado em análises estatísticas, o número de casos e óbitos de COVID-19 que ocorrerá no Brasil e no estado de Mato Grosso poderá instrumentalizar os gestores para o planejamento de ações estratégicas de prevenção, monitoramento e controle da pandemia.

Palavras-chave: Coronavírus. COVID-19. Previsão. Incidência. Epidemiologia.

\begin{abstract}
Objective: to predict the epidemiological trend of COVID-19 cases and deaths in Brazil and Mato Grosso state using the double exponential statistical model. Methods: in order to carry out this work, data on cases and deaths from COVID-19 provided by the Ministry of Health of Brazil and the State Secretariat of Health of Mato Grosso were used and to make the predictions with these data, the time series statistical technique was used, considering the double exponential model and the MINITAB V 17 program was used for data processing. Results: the results show that the values adjusted by the double exponential transformed model of cases and deaths are very close to the observed values of COVID-19 in Brazil and Mato Grosso, in the period considered, indicating that this model is suitable for the data under study, which was verified by predicting some values with their respective $95 \%$ confidence intervals. Conclusions: the possibility of estimating, based on statistical analysis, the number of cases and deaths of COVID-19 that will occur in Brazil and in the state of Mato Grosso, may instrumentalize the managers for the planning of strategic actions for prevention, monitoring and control of pandemic.
\end{abstract}

Keywords: Coronavirus. COVID-19. Prediction. Incidence. epidemiology.

\section{INTRODUÇÃO}

Os coronavírus foram identificados, pela primeira vez, em 1937, porém, apenas em 2002, estiveram associados à Síndrome Respiratória Aguda Grave (SARS) e à Síndrome Respiratória do Oriente Médio (MERS) em 2014. Os subtipos virais adaptados aos seres humanos saltaram de animais como roedores, morcegos e camelos $^{1,2}$. Em decorrência das probabilidades de mutações adaptativas e características do receptor ACE2, responsável pela ligação a 2019-nCoV, há evidências de que a origem do novo coronavírus (COVID-19) é proveniente do morcego $^{3}$.

Sua emergência no fim de dezembro de 2019, na cidade de Wuhan, China, surpreendeu o mundo, expandindo-se e tornando-se uma das mais impactantes pandemias da atualidade ${ }^{4}$, com registro de 3.672 .238 casos e 254.045 óbitos no mundo ${ }^{5}$ e 135.106 casos com 9.146 mortes no Brasil até 07 de maio de $2020^{6}$.

A alta transmissibilidade ${ }^{7}$ associada às várias formas de contágio até então descritas como contato direto, por saliva, aerossol, fezes e urina ${ }^{8}$, constituem alto risco de disseminação para população. Em decorrência da inexistência de medicamentos e vacina disponíveis, as estratégias de prevenção e controle da doença são as estratégias essenciais neste momento da pandemia ${ }^{9}$.

Em todo o mundo, as medidas de isolamento social foram adotadas e conseguiram reduzir as infecções, além de favorecer o atendimento médico em decorrência do achatamento da curva epidêmica ${ }^{10}$. No entanto, é fundamental que os serviços de saúde locais possam antever os riscos para que, assim, possam gerenciar as ações preventivas e assistenciais ${ }^{11}$. Nesse 
contexto, a modelagem estatística é útil, pois atua na previsão de possibilidades e gravidade da pandemia, fornecendo informações importantes para a ação ${ }^{12}$.

Trabalhos anteriores sobre predição de casos em faces de epidemias de influenza e zika apontaram sobre a importância dessa ação como instrumento essencial para o direcionamento das ações preventivas ${ }^{13,14}$. Desde a difusão do COVID-19 pelo mundo, essa ferramenta foi utilizada em perspectivas globais ${ }^{15}$. No Brasil, é descrito em artigo produzido pela equipe do Ministério da Saúde, que modelos matemáticos preveem que o vírus estará circulando até setembro com pico em abril e maio; porém, não foram identificadas publicações que detalhem tais apontamentos $^{16}$.

Diante desse cenário, objetivou-se prever a tendência epidemiológica do número de casos no Brasile, especificamente, em Mato Grosso e de óbitos brasileiros por meio do modelo estatístico duplo exponencial.

\section{MÉTODOS}

\section{Descrição e análise estatística dos dados}

Para a realização deste trabalho, foram utilizados dados do Ministério da Saúde (MS) do Brasil e da Secretaria de Estado de Saúde de Mato Grosso (SES-MT), que gera e disponibiliza, via Internet, dados diários do número de casos e óbitos no país. Os dados fornecidos pelo MS possibilitam uma visão geral da situação diária do coronavírus no país, em um determinado período.

Para prever os casos e óbitos do novo coronavírus no Brasil e, especificamente, em Mato Grosso, foi utilizada a técnica estatística de séries temporais, considerando o modelo exponencial duplo. E, para o processamento dos dados, foi utilizado o programa computacional MINITAB V 17.

Neste estudo, foram consideradas duas variáveis dependentes: o número de casos e óbitos do COVID-19 no Brasil, e a variável independente foi data de divulgação da ocorrência do óbito. Para Mato Grosso, foi considerado apenas o número de casos de COVID-19, pois esse registro ainda é muito baixo.

\section{Modelagem e análise estatística dos dados}

Para prever os casos e óbitos do novo coronavírus, foi utilizada a técnica estatística de séries temporais, considerando que os dados estão ordenados no tempo. Antes de modelar a série temporal, construiu-se um gráfico, para visualizar o comportamento geral da série temporal de COVID-19 no Brasil e no estado do Mato Grosso e identificar as tendências seculares, os movimentos cíclicos e as sazonalidades ${ }^{17}$.

Ao supor que a tendência pode ser representada por um polinômio de baixo grau, isso implica que utilizamos todas as observações $Z_{t^{\prime}} t=1,2, \cdots, n$, para estimar o polinômio, que representará $\mathrm{T}_{\mathrm{t}}$ sobre todo o intervalo de tempo considerado. Nesse caso, os métodos de amortecimento são os métodos de previsão bastante utilizados, devido à sua facilidade de implementação computacional e à sua qualidade das previsões geradas. Entre os métodos de amortecimento mais utilizados, estão a Suavização Exponencial Simples (SES), Holt e Winters ${ }^{18}$.

Quando o modelo exponencial simples é aplicado na previsão de séries temporais que apresentam tendência entre as observações passadas, os valores previstos podem superestimar ou subestimar os valores reais. Assim, a precisão das previsões pode ser afetada ${ }^{18}$.

Quando os dados da série apresentam uma tendência, é preciso considerar um modelo com tendência. Neste caso, um dos métodos utilizados é a suavização exponencial de Holt ou a suavização dupla exponencial. Esse método utiliza um componente de nível e outro de tendência para cada período de tempo considerado. Assim, essa série temporal é composta, localmente, da soma de nível, tendência e resíduo aleatório com média zero e variância constante $\left(\theta^{2}{ }_{a}\right)$, a qual pode ser escrita da seguinte maneira:

$$
Z_{t}=\mu_{t}+T_{t}+a_{t}
$$

para $t=1,2, \ldots, n$

Os valores do nível e da tendência da série serão estimados por:

e

$$
\bar{Z}_{t}=\alpha\left(Z_{t}\right)+\left(1-\alpha \gamma \bar{Z}_{t-1}+\hat{T}_{t-1}\right)
$$

$$
\hat{T}_{t}=\gamma\left(\bar{Z}_{t}-\bar{Z}_{t-1}\right)+(1-\gamma) \hat{T}_{t-1}
$$

respectivamente, nas quais e são as constantes de alisamento ou suavização, para $t=2, \ldots, n^{18}$.

Assim, a previsão para o valor $\mathrm{Z}_{\mathrm{t}+\mathrm{h}^{\prime}}$, com origem em $t$ é dada por: $\quad \hat{Z}_{t}(h)=\bar{Z}_{t}+h \hat{T}_{t} \forall h>0$

isto é, a previsão é feita, adicionando-se ao valor básico $\left(\bar{Z}_{t}\right)$ a tendência multiplicada pelo número de passos à frente que se deseja prever $(h)$.

Medidas de adequação do modelo do ajustado: em geral, existem três medidas de adequação do modelo ajustado: o erro de porcentagem absoluto médio (EPAM), o erro absoluto médio (EAM) e o erro quadrático médio (EQM) para cada um dos métodos simples de previsão e suavização. Para todas as três medidas, quanto menor o valor, melhor o ajuste do modelo. Devem-se utilizar essas estatísticas para comparar os ajustes dos diferentes métodos de previsão e suavização. Embora existam essas três medidas na prática, em geral, é utilizada a EPAM ou MAPE (Mean Absolute Percentage Error). Por isso, neste trabalho, somente será apresentada fórmula para esse valor. As outras fórmulas podem ser encontradas nos livros séries temporais; ver, por exemplo, Morettin e Toloi ${ }^{18}$. No 
entanto, essas medidas devem ser utilizadas após uma análise residual.

O erro de porcentagem absoluto médio (EPAM): mede a adequação dos valores ajustados da série temporal. Essa medida expressa a adequação do modelo em porcentagem:

$$
E P A M=\frac{\sum_{i=1}^{n}\left|\left(z_{t}-\hat{z}_{t}\right) / z_{t}\right|}{n} \times 100
$$

para $z_{t} \neq 0$, na qual $z_{t}$ é o valor atual no tempo $t, \hat{Z}_{t}$ é o valor ajustado e $n$ é o número de observações.

Este estudo respeitou todos os aspectos éticos em pesquisa, conforme Declaração de Helsinki e Resolução 466/2019 do Conselho Nacional de Saúde (CNS/Brasil), e não foi submetido para apreciação em comitê de ética por se tratar de dados disponíveis on line em boletins diários de informações sobre a doença no Brasil e no estado de Mato Grosso.

\section{RESULTADOS}

Inicialmente, foi realizada uma análise descritiva dos dados, por meio de um gráfico para identificar o comportamento da curva dos casos e dos óbitos do COVID-19 no Brasil, no período de 26 de fevereiro a 07 de maio 2020 para os casos, e de 17 de março a 07 de maio para os óbitos, e, em Mato Grosso, no período de 14 de março a 07 de maio de 2020. Esses valores são representados nas figuras 1 e 2 e diferenciam-se, pois o primeiro registro no Brasil e no estado de Mato Grosso foram em datas distintas.

Na figura 1, observa-se um comportamento exponencial dos números de casos e óbitos no Brasil no período analisado. Nessa figura, também são apresentados os valores dos casos

Figura 1. gráfico do número de casos e óbitos com valores ajustados pelo modelo duplo exponencial transformado, por data no período de 26 de fevereiro de 2020 a 07 de maio de 2020 e do período de 17 de março a 07 de maio, respectivamente.
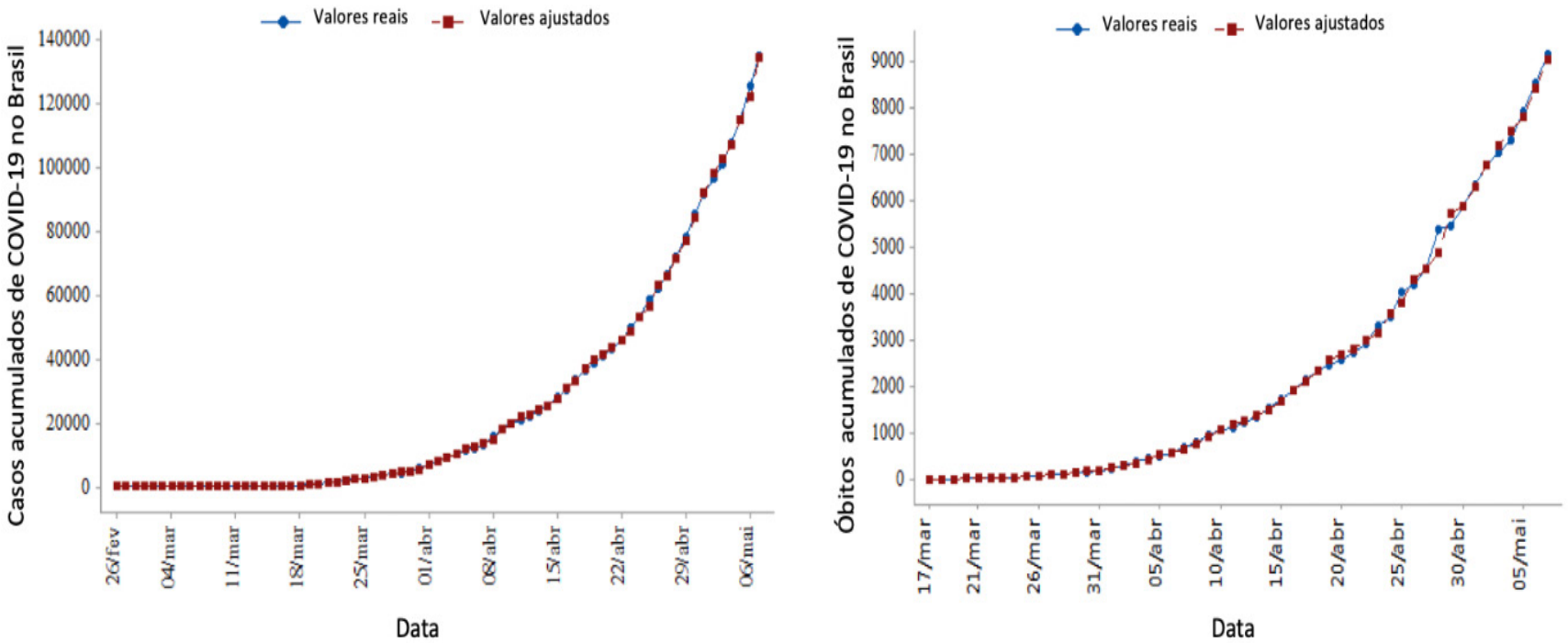

Figura 2. gráfico do número de casos em Mato Grosso com valores ajustados pelo modelo duplo exponencial transformado, por data no período de 14 de março de 2020 a 07 de maio de 2020.

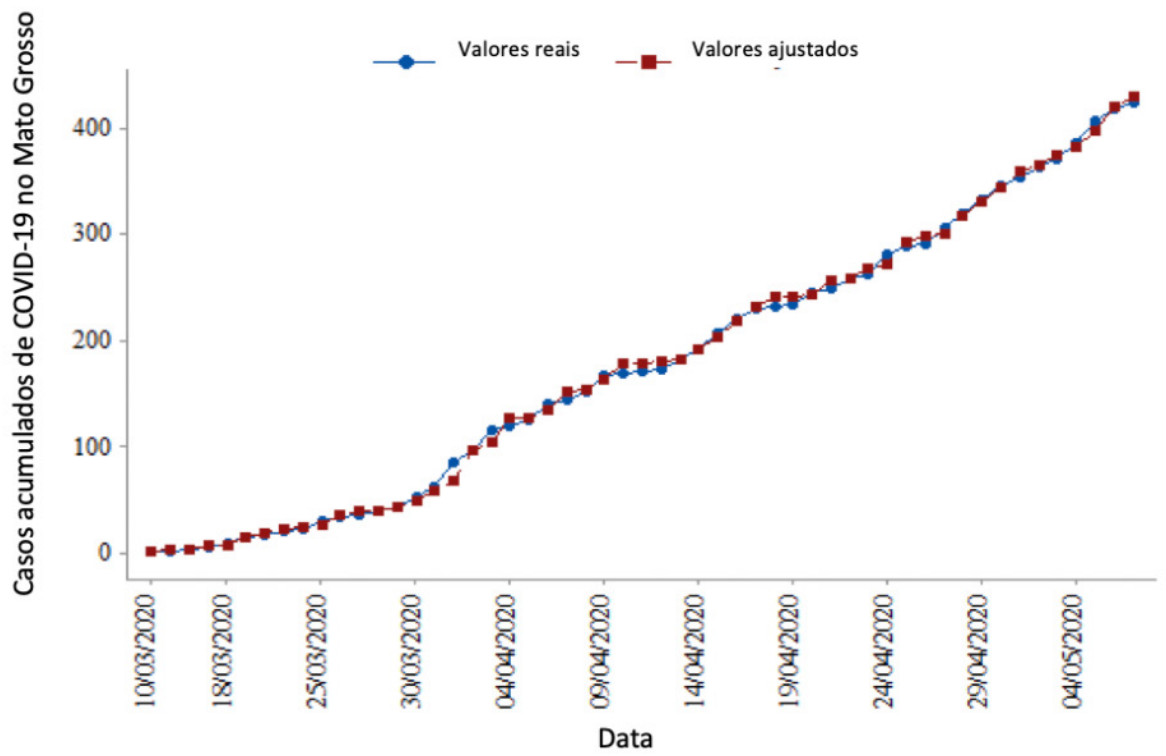


(valores reais) e ajustados pelo modelo exponencial duplo. Cabe destacar que, após a análise residual, foi necessária uma transformação nos dados. Nessa situação, a transformação indicada pelo método de Box e Cox19 foi dos casos elevados a $0,2036256154\left(y^{*}=y^{(0,2036256154)}\right)$.

Ao comparar os valores ajustados pelo modelo exponencial duplo dos casos, os dados transformados com valores reais dos casos de COVID-19 no Brasil, no período considerado, estão muito próximos, o que indica que o modelo é adequado para os dados considerados.

Quanto aos valores dos óbitos observados (valores reais) e ajustados pelo modelo exponencial duplo (figura 1); neste modelo, também depois da análise residual, foi preciso fazer uma transformação pelo método de Box e $\operatorname{Cox}^{19}$ para os dados do número de casos do CIVD-19. Nessa situação, a transformação indicada por esse método foi de 0,3157006187 $\left(y^{*}=y^{(0,3157006187)}\right)$. para os óbitos. É evidenciado que os valores ajustados pelo modelo exponencial duplo transformado dos óbitos estão muito próximos dos valores observados dos óbitos de COVID-19 no Brasil, no período considerado, indicando, também, que esse modelo é adequado para os dados em estudo.

Quanto aos valores de alfa e gama, ajustados pelo modelo exponencial duplo para o número de casos de COVID-19 no Brasil, os dados transformados foram 1,21598 ( $\alpha=1,21598)$ e $0,15083$ ( $y=0,15083)$, respectivamente para alfa e gama e o valor da $E M A=1,77 \%$. Já os valores de alfa e gama ajustados pelo modelo exponencial duplo transformado para o número de óbitos por COVID-19 no Brasil foram 0,902613 ( $\alpha=0,902613$ ) e 0,033918 ( $y=0,033918)$, respectivamente para alfa e gama e o valor da $E M A=1,74 \%$.

O modelo duplo exponencial dos dados transformados ( $\left.y^{*}=y^{(0,1769351627)}\right)$ pode ser utilizado para fazer previsões do número de casos de COVID-19 no Brasil, bem como os dados transformados $\left(y^{* *}=y^{(0,3049388095)}\right)$ podem ser utilizados para fazer previsões do número de óbitos por COVID-19 no período crítico previsto pelos profissionais de saúde. Obviamente que, após a transformação, é preciso retornar à escala original dos dados. As previsões e os intervalos de $95 \%$ de confiança para os próximos 14 dias são apresentados na tabela 1.

Tabela 1. números estimados de casos e óbitos de COVID-19 no Brasil e intervalo de confiança a 95\% para os próximos 7 dias no Brasil, com o modelo duplo exponencial dos dados transformados.

\begin{tabular}{lrrrr}
\hline Data & $\begin{array}{r}\text { Número de } \\
\text { casos estimados }\end{array}$ & $\begin{array}{r}\text { Intervalo de confiança } \\
\text { de 95\% }\end{array}$ & $\begin{array}{r}\text { Número de } \\
\text { óbitos estimados }\end{array}$ & $\begin{array}{r}\text { Intervalo de confiança } \\
\text { de 95\% }\end{array}$ \\
\hline $08 /$ mai & 144.342 & {$[136885 ; 152126]$} & 9.691 & {$[9302 ; 10092]$} \\
$09 /$ mai & 153.904 & {$[140244 ; 168602]$} & 10.269 & {$[9692 ; 10869]$} \\
$10 /$ mai & 163.963 & {$[143499 ; 186686]$} & 10.869 & {$[10082 ; 11698]$} \\
$11 /$ mai & 174.539 & {$[146762 ; 206341]$} & 11.494 & {$[10478 ; 12574]$} \\
$12 /$ mai & 185.643 & {$[150065 ; 227645]$} & 12.142 & {$[10882 ; 13498]$} \\
$13 /$ mai & 197.311 & {$[153413 ; 250675]$} & 12.814 & {$[11296 ; 14468]$} \\
$14 /$ mai & 209.555 & {$[156820 ; 275545]$} & 13.512 & {$[11718 ; 15486]$} \\
\hline
\end{tabular}

Para o estado de Mato Grosso, também se observa, na figura 2, um comportamento exponencial dos números de casos no período de 14/3 ao 7/5. Nesta mesma figura, são apresentados os valores dos casos (valores reais), que são ajustados pelo modelo exponencial duplo. Cabe destacar que, após a análise residual, foi necessária uma transformação nos dados pelo método de Box e Cox19, dos casos elevados a 0,5 $\left(y^{*}=y^{(0,5)}\right)$.
Quanto aos valores de alfa e gama ajustados pelo modelo exponencial duplo para o número de casos de COVID-19 no estado de Mato Grosso, os dados transformados foram 11,16036 $(\alpha=1,16036)$ e $0,03955(y=0,03955)$, respectivamente para alfa e gama, e o valor da $E M A=4,42$.. As previsões e os intervalos de $95 \%$ de confiança para os próximos 7 dias são apresentados na tabela 2.

Tabela 2. número estimado de casos de COVID-19 no Estado de Mato Grosso e intervalo de confiança a $95 \%$ para os próximos 7 dias, com o modelo duplo exponencial dos dados transformados.

\begin{tabular}{lrr}
\hline Data & $\begin{array}{r}\text { Número de casos } \\
\text { estimados }\end{array}$ & $\begin{array}{r}\text { Intervalo de confiança } \\
\text { de } 95 \%\end{array}$ \\
\hline $08 /$ mai & 436 & {$[421 ; 451]$} \\
$09 /$ mai & 448 & {$[423 ; 474]$} \\
$10 /$ mai & 461 & {$[424 ; 498]$} \\
$11 /$ mai & 473 & {$[425 ; 522]$} \\
$12 /$ mai & 485 & {$[427 ; 547]$} \\
$13 /$ mai & 498 & {$[428 ; 572]$} \\
$14 /$ mai & 511 & {$[429 ; 597]$} \\
\hline
\end{tabular}




\section{DISCUSSÃO}

A análise de tendências temporais de casos e óbitos de COVID-19, embora seja algo recente na saúde pública, é uma técnica importante, pois pode prever as tendências epidemiológicas desta pandemia. Utilizado com êxito na China ${ }^{20}$ e Irã ${ }^{11}$ e em previsões para diversos países do mundo ${ }^{15}$, tem como função estabelecer parâmetros que direcionam as ações governamentais na redução da transmissão da doença ${ }^{21}$, por meio de ações assertivas ajustadas às realidades locais.

No presente estudo, assim como em pesquisas conduzidas em países afetados anteriormente ao Brasil $^{22}$, foi evidenciado um aumento muito rápido do número de casos e óbitos de COVID-19, com R0 estimado de 2,6 a 4,1. Os primeiros 60 dias de circulação da doença apresentam disseminação mais rápida, possivelmente associada à alta transmissibilidade, à adoção tardia de medidas de distanciamento social, ao grande número de assintomáticos/subnotificação e ao período de incubação de até 14 dias $^{23}$. 0 aumento rápido desses números ordenados no tempo corrobora a utilização do modelo estatístico duplo exponencial, inclusive, por o Brasil estar no 62임 da primeira confirmação de COVID-19 no território nacional e Mato Grosso, no 53 이. dia.

Cabe ressaltar que a adoção precoce de ações governamentais como detecção e isolamento precoce dos casos, distanciamento social e redução de interação da população, monitoramento das fronteiras e ampliação de higiene pessoal podem reduzir a disseminação e, assim, modificar o comportamento da doença ${ }^{24}$. Adicionalmente, ações opostas às descritas acima podem ocasionar a explosão de casos e, consequentemente, dos óbitos.

Após a infecção pelo COVID-19, ocorre ligação do vírus às células alvo com receptores da angiotensina 2 (ACE2), favorecendo a replicação viral. Esse receptor está presente em diferentes órgãos; porém, o maior comprometimento relatado é no pulmão, com dano alveolar difuso avançado e pneumonia ${ }^{2,4,25}$ que, em alguns casos, evoluem para o óbito. Em estudos conduzidos na China ${ }^{26}$ e Itália ${ }^{27}$, identificaram que os óbitos estão relacionados com a idade avançada, o fato de ser portador de doença crônica, apresentar uma infecção secundária/coinfecção e ter indicadores inflamatórios elevados no sangue.

Nesse contexto, a taxa de mortalidade por COVID-19 foi descrita com intervalos amplos em estudos preliminares $(0,3-$ $15 \%)^{28}$, sendo justificado pela dificuldade na identificação dos infectados, seja pela grande quantidade de assintomáticos, seja por falta de acesso ao diagnóstico laboratorial em quantidade e tempo oportuno ${ }^{29}$.Predizer os óbitos é fundamental para direcionar as ações dos serviços de saúde, pois sabe-se que essa parcela de pacientes necessitou de hospitalização em alta complexidade, e a estruturação desse serviço perpassa pela previsão de ocupação dos leitos. Ressalta-se que a previsão dos óbitos em Mato Grosso não foi realizada, tendo em vista o reduzido número desse evento no estado (4 óbitos no primeiro mês da doença).

No entanto, na utilização do modelo duplo exponencial para os dados considerados, foi necessário fazer uma transformação de $\mathrm{BOX}$ e $\mathrm{COX}^{19}$ para os dados do número de casos e óbitos do COVID-19, para estabilizar a variância, devido à grande variabilidade desses dados, a fim de que o modelo fosse adequado, pois as previsões devem caracterizar-se pela acurácia de seus resultados, pela simplicidade dos métodos empregados e, sobretudo, pela confiabilidade estatística dos modelos empregados para gerar as previsões ${ }^{18}$.

O modelo utilizado no presente estudo com os dados dos casos e dos óbitos transformados foi, estatisticamente, adequado, para fazer as previsões dos dois eventos analisados, atendendo, assim, aos critérios de validação com valores reais e, além disso, atendendo à precisão requerida estatisticamente. Estudos conduzidos em epidemias de dengue apresentaram sucesso e sugeriram que os modelos estatísticos de predição fossem ferramentas importantes na gestão de epidemias das doenças infecciosas ${ }^{30}$. Cabe ressaltar que a realização de análises em curto período de tempo é uma ferramenta importante, pois, como a pandemia é dinâmica e com comportamento inesperado, é fundamental que essa análise ocorra a cada 7 dias e auxilie no monitoramento e avaliação, redirecionando as ações e ajustando-as à nova realidade.

\section{CONCLUSÃO}

O estudo da previsão da tendência epidemiológica por análise de séries temporais pode ser um instrumento adequado para a avaliação dos casos e óbitos do COVID-19. A utilização dessa técnica de análises, considerando o modelo estatístico duplo exponencial, permite avaliar e prever o comportamento, do número casos ébitos dessa doença no Brasil e, especificamente, em Mato Grosso, pois essa técnica fornece valores informativos que podem auxiliar os gestores no planejamento de ações estratégicas de prevenção, monitoramento e controle da pandemia e, como proposta, a reavaliação em curto período pode ajustar as necessidades atuais e potencializar as ações assertivas que contribuirão com a redução de casos e óbitos.

\section{CONTRIBUIÇÃO DOS AUTORES}

MME, ECO, JSM, RDD, ACPTT contribuíram para o delineamento do estudo, a redação do manuscrito, a análise e compilação dos dados, a revisão crítica e a busca na literatura, bem como contribuiu para o delineamento de tabelas e figuras. 


\section{REFERÊNCIAS}

1. Drosten C, Gunther S, Periser W, Van der Welf S, Brodth HR, Becker S, et al. Identification of a novel coronavirus in patients with severe acute respiratory syndrome. N. Engl. J. Med [Internet]. 2003 Maio [acesso 2020 Abr 14]; 348(20): 1967-1976. Disponível em: https://www.ncbi.nlm.nih.gov/pubmed/12690091.

2. Su S, Wong G, Shi W, Liu J, Lai ACK, Zhou J, et al. Epidemiology, genetic recombination, and pathogenesis of coronaviruses. Trends Microbiol [Internet]. 2016 Jun [acesso 2020 Abr 14]; 24(6): 490-502. Disponível em: https://www. ncbi.nlm.nih.gov/pubmed/27012512.

3. Sun J, He WT, Wang L, Lai A, Ji X, Zhai X, et al. Covid-19: Epidemiology, Evolution, and Cross-Disciplinary Perspectives. Trends Molec Medicine [Internet]. 2020 [acesso 2020 Abr 14]; 2020 Mar; 1-13. Disponível em: https:// www.cell.com/action/showPdf?pii=S1471-4914\%2820\%2930065-4.

4. Li Q, Guan X, Wu P, Wang X, Zhou L, Tong Y, et al. Early transmission dynamics in Wuhan, China, of novel coronavirus-infected pneumonia. N Engl J Med [Internet]. 2020 [acesso 2020 Abr 14]; 382: 1199-1207. Disponível em: https:// www.nejm.org/doi/full/10.1056/NEJMoa2001316.

5. World Health Organization. Coronavirus disease 2019 (COVID-19) SituationReport-108. Geneva: WHO; 2020. Disponível em: https://www.who. int/emergencies/diseases/novel-coronavirus-2019/situation-reports/.

6. Ministério da Saúde [BR]. Centro de operações de emergência em Saúde Pública. Coronavírus COVID-19: Boletim epidemiológico diário. Brasília: Ministério da Saúde; 2020. Disponível em: https://www.saude.gov.br/images/ pdf/2020/April/10/10.04.2020-COVID.pdf.

7. Chan JFW, Yuan S, Kok KH, To KKW, Chu H, Yang J, et al. A familial cluster of pneumonia associated with the 2019 novel coronavirus indicating personto-person transmission: a study of a family cluster. Lancet [Internet]. 2020 [acesso 2020 Abr 14]; 395(10223): 514-523. 2020. Disponível em: https://www. thelancet.com/journals/lancet/article/PIIS0140-6736(20)30154-9/fulltext.

8. Wang LS, Wang YR, Ye DW, Liu QQ. A review of the 2019 Novel Coronavirus (COVID-19) based on current evidence. Int J Antimicrob Agents [Internet]. 2020 Mar [acesso 2020 Abr 14]; 105948. 2020. Disponível em: https://www. sciencedirect.com/science/article/pii/S0924857920300984.

9. Wilder-Smith A, Freedman DO. Isolation, quarantine, social distancing and community containment: pivotal role for old-style public health measures in the novel coronavirus (2019-nCoV) outbreak. J Travel Med [Internet]. $2020 \mathrm{Mar}$ [acesso $2020 \mathrm{Abr} 14]$; 27(2): 1-4. Disponível em: https://academic.oup.com/ jtm/article/27/2/taaa020/5735321.

10. Chinazzi M, Davis JT, Ajelli M, Gioannini C, Litvinova M, Merler S, et al The effect of travel restrictions on the spread of the 2019 novel coronavirus (COVID-19) outbreak. Science [Internet]. 2020 [acesso 2020 Abr 14]; 1-11. Disponível em: https://science.sciencemag.org/content/early/2020/03/05/ science.aba9757.

11. Ayyoubzadeh SM, Ayyoubzadeh SM, Zahedi H, et al. Predicting COVID-19 incidence using Google Trends and data mining techniques: A pilot study in Iran. JMIR Public Health and Surveill [Internet]. 2020 Mar [acesso 2020 Abr 14]; 6(2). 2020. Disponível em: https://www.ncbi.nlm.nih.gov/pubmed/32234709.

12. Santillana M, Nguyen AT, Dredze M, Paul MJ, Nsoesie EO, Brownstein, JS. Combining search, social media, and traditional data sources to improve influenza surveillance. PLoS [Internet]. 2015 Out [acesso 2020 Abr 14]; 11(10). Disponível em: https://journals.plos.org/ploscompbiol/article?id=10.1371/ journal.pcbi.1004513.

13. McGough SF, Brownstein JS, Hawkins JB, Santillana M. Forecasting Zika incidence in the 2016 Latin America outbreak combining traditional disease surveillance with search, social media, and news report data. PLoS [Internet]. 2017 Jan [acesso 2020 Abr 14];11 (1):e0005295. Disponível em: https:// journals.plos.org/plosntds/article?id=10.1371/journal.pntd.0005295.
14. Niakan Kalhori SR, Ghazisaeedi M, Azizi R, Naserpour A. Studying the influence of mass media and environmental factors on influenza virus transmission in the US Midwest. Public health [Internet]. 2019 [acesso $2020 \mathrm{Abr}$ 14];170:17-22. Disponível em: https://www.sciencedirect.com/science/article/ abs/pii/S0033350619300307?via\%3Dihub.

15. ang B, Wang X, Li Q, Bragazzi NL, Tang S, Xiao $Y$, et al. Estimation of the Transmission Risk of the 2019-nCoV and Its Implication for Public Health Interventions. J Clin Med [Internet]. 2020 Fev [acesso 2020 Abr 14]; 9(2): E462. Disponível em: https://www.mdpi.com/2077-0383/9/2/462.

16. Croda J, Oliveira WK, Frutuoso RL, Mandetta LH, Baia-da-Silva DC, Sousa JDB, et al. COVID-19 in Brazil: advantages of a socialized unified health system and preparation to cotain cases. Rev Soc Bras Med Trop [Internet]. 2020 Abr [acesso 2020 Abr 14];53(e20200167):1-6. Disponível em: https://preprints.scielo.org/ index.php/scielo/preprint/view/7/14.

17. Espinosa MM, Prado SM, Ghellere M. Uso do modelo SARIMA na previsão do número de focos de calor para os meses de junho a outubro no Estado de Mato Grosso. Ciência e Natura [Internet]. 2010 acesso 2018 Jun 01];32(2):721. Disponível em: https://periodicos.ufsm.br/cienciaenatura/article/view/9482.

18. Morettin PA, Toloi CMC. Análise de séries temporais. São Paulo: Edgar BlüCher; 2004.

19. Box GEP; Cox DR. An analysis of transformations. J. Royal Soc [Internet]. 1964 [citado em 19 de setembro de 2019];26:211-252. Disponível em: http:// links.jstor.org/sici?sici=0035-9246\%281964\%2926\%3A2\%3C211\%3AAAOT\%3E 2.0.CO\%3B2-6.

20. Wu JT, Leung K, Leung GM. Nowcasting and forecasting the potential domestic and international spread of the 2019-nCoV outbreak originating in Wuhan, China: a modelling study. Lancet [Internet]. 2020 [acesso 2020 Abr 14];395(10225): 689-697. Disponível em: https://www.thelancet.com/ journals/lancet/article/PIIS0140-6736(20)30260-9/fulltext.

21. Fang Y, Nie Y, Penny M. Transmission dynamics of the COVID-19 outbreak and effectiveness 265 of government interventions: A data-driven analysis. J Med Virol [Internet]. 2020 Mar [acesso 2020 Abr 14]; 1-15. Disponível em: https://onlinelibrary.wiley.com/doi/full/10.1002/jmv.25750.

22. Liu Y, Gayle AA, Wilder-Smith A, Rocklöv J. The reproductive number of COVID-19 is higher 279 compared to SARS coronavirus. J Travel Med [Internet]. 2020 Mar [acesso 2020 Abr 14]; 27(2):1-4. Disponível em: https://www.ncbi. nlm.nih.gov/pmc/articles/PMC7074654/pdf/taaa021.pdf.

23. Guan WJ, Ni ZY, Hu Y, Liang WH, Ou CQ, He JX, et al. Clinical Characteristics of Coronavirus 282 Disease 2019 in China. N Engl J Med [Internet]. 2020 Fev [acesso 2020 Abr 14];1-13. Disponível em: https://www.ncbi.nlm.nih.gov/pmc/ articles/PMC7092819/.

24. Aleta A, Moreno Y. Evaluation of the potential incidence of COVID-19 and effectiveness of 301 contention measures in Spain: a data-driven approach. MedRxiv [Internet]. 2020 [acesso 2020 Abr 14]; 1-12. Disponível em: https:// www.medrxiv.org/content/10.1101/2020.03.01.20029801v2.full.pdf+html.

25. Xu Z, Shi L, Wang YJ, Zhang JY, Huang L, Zhang C, et al. Pathological findings of COVID-19 associated with acute respiratory distress syndrome. Lancet Respir Med [Internet]. 2020 Abr [acesso 2020 Abr 14]; 8(4): 420-422. Disponível em: https://www.thelancet.com/journals/lancet/article/PIIS22132600(20)30076-X/fulltext.

26. Ruan Q, Yang K, Wang W, Zhang J, Huang L, Zhang C, et al. Clinical predictors of mortality due to COVID-19 based on an analysis of data of 150 patients from Wuhan, China. Intensive Care Med [Internet]. 2020 Mar [acesso 2020 Abr 14];13. Disponível em: https://www.ncbi.nlm.nih.gov/pmc/articles/PMC7080116/.

27. Onder G, Rezza G, Brusaferro S. Case-Fatality Rate and Characteristics of 
Patients Dying in Relation to COVID-19 in Italy. JAMA [Internet]. 2020 Mar [acesso $2020 \mathrm{Abr}$ 14];E1-E2. Disponível em: https://jamanetwork.com/ journals/jama/fullarticle/2763667.

28. Rajgor DD, Lee MH, Archuleta S, Bagdasarian N. The many estimates of the COVID-19 case fatality rate. Lancet Infect Dis [Internet]. 2020 Mar [acesso 2020 Abr 14]; 3099(20): 30244-9. Disponível em: https://www.thelancet.com/ action/showPdf?pii=S1473-3099\%2820\%2930244-9.
29. Bai, Y, Yao L, Wei T, Tian F, Jin DY, Chen J. Presumed Asymptomatic Carrier Transmission of COVID-19. JAMA [Internet]. 2020 Fev [acesso 2020 Abr 14] 323(14): 1406-1407. Disponível em: https://jamanetwork.com/journals/jama/ fullarticle/2762028.

30. Buczak L, Baugher B, Moniz LJ, Bagley T, Babin SM, Guven E. Ensemble method for dengue prediction. PloS [Internet]. 2018 Jan [acesso $2020 \mathrm{Abr}$ 14]; 13(1): e0189988. Disponível em: https://journals.plos.org/plosone/ article?id=10.1371/journal. pone. 0189988 .

Espinosa MM, Oliveira EC, Melo JS, Damaceno RD, Terças-Trettel ACP. Predição de casos e óbitos de COVID-19 em Mato Grosso e no Brasil. J Health Biol Sci. 2020 J; 8(1):1-7. 